\title{
A CLASS OF INFINITELY CONNECTED DOMAINS AND THE CORONA

\author{
BY
}

W. M. DEEB

\begin{abstract}
Let $D$ be a bounded domain in the complex plane. Let $H^{\infty}(D)$ be the Banach algebra of bounded analytic functions on $D$. The corona problem asks whether $D$ is weak* dense in the space $\Re(D)$ of maximal ideals of $H^{\infty}(D)$. Carleson [3] proved that the open unit disc $\Delta_{0}$ is dense in $\Re\left(\Delta_{0}\right)$. Stout [9] extended Carleson's result to finitely connected domains. Behrens [2] found a class of infinitely connected domains for which the corona problem has an affirmative answer.

In this paper we will use Behrens' idea to extend the results to more general domains. See [11] for further extensions and applications of these techniques.
\end{abstract}

Introduction. By a $\Delta$-domain we mean a domain $D$ obtained from the open unit disc $\Delta_{0}$ by deleting the origin and a sequence of disjoint closed discs $\Delta_{n}=\Delta\left(c_{n}, r_{n}\right)=\left\{z:\left|z-c_{n}\right| \leqslant r_{n}\right\}$ with $c_{n} \rightarrow 0$. Under theadditional hypothesis $\sum r_{n} /\left|c_{n}\right|<\infty$, Zalcman showed in [10] that there is a distinguished homomorphism in $\Re(D)$ defined by

$$
\phi_{0}(f)=\frac{1}{2 \pi i} \int_{\partial D} \frac{f(\xi)}{\xi} d \xi .
$$

The distinguished homomorphism is always adherent to $D$ [7]. Behrens showed that if the corona fails for any domain it fails for a $\Delta$-domain. He also showed that if there are numbers $R_{n}>r_{n}$ such that $\Sigma r_{n} / R_{n}<\infty$ and the discs $D_{n}=\Delta\left(c_{n}, R_{n}\right)$ are disjoint, then $D$ is dense in $\Re(D)$. To prove this Behrens constructed an isomorphism of $H^{\infty}(D)$ into $H^{\infty}\left(\Delta_{0} \times N\right)$, the algebra of bounded functions which are analytic on each slice of $\Delta_{0} \times N$, where $N$ is the nonnegative integers. He then used the fact that $\Delta_{0} \times N$ is dense in $\mathfrak{R}\left(\Delta_{0} \times N\right)$ [1], to obtain $D$ is dense in $\Re(D)$. In this paper we will use Behrens' idea to extend the results to more general domains.

Notation and statement of results. Throughout we assume that there exist numbers $R_{n}>r_{n}$ such that the discs $D_{n}=\Delta\left(c_{n}, R_{n}\right)$ are disjoint and such that $r_{n} / R_{n} \rightarrow 0$. For notational convenience let $c_{0}=0, r_{0}=R_{0}=1$. Let $E_{0}(z)=z$ and $E_{n}(z)=r_{n} /\left(z-c_{n}\right)$, for $z \in \Delta_{n}^{c}=\mathbf{C} \backslash \Delta_{n}, n=1,2, \ldots$ Choose a

Received by the editors December 10, 1974 and, in revised form, January 29, 1976.

AMS (MOS) subject classifications (1970). Primary 46J15, 30A98.

O American Mathematical Society 1977 
sequence of positive integers $\left\{k_{n}\right\}$ such that $\Sigma\left(r_{n} / R_{n}\right)^{k_{n}}<\infty$. Let $L_{n}(z)=$ $E_{n}(z)^{k_{n}}-E_{n}(0)^{k_{n}}$ and $L(z)=\Sigma L_{n}(z)$. Choose $S_{n}=\sqrt{r_{n} R_{n}}$ so that $r_{n} / S_{n} \rightarrow 0$ and $S_{n} / R_{n} \rightarrow 0$, and let $B_{n}=\Delta\left(c_{n}, S_{n}\right)$. Let

$$
X=\Re\left(\Delta_{0} \times N\right) \backslash\left[\bigcup_{n} \Re\left(\Delta_{0}\right) \times\{n\}\right] .
$$

For $f \in H^{\infty}(D)$ let

$$
a_{n}(f)=\frac{1}{2 \pi i} \int_{\partial \Delta_{n}} \frac{f(\zeta)}{\zeta-c_{n}} d \zeta \text { and }\left(P_{n} f\right)(z)=\frac{1}{2 \pi i} \int_{\partial \Delta_{n}} \frac{f(\zeta)}{\zeta-z} d \zeta \text {, }
$$

where $z \in \Delta_{0}$ if $n=0$ and $z \in \Delta_{n}^{c}$ if $n=1,2,3, \ldots$ Let $\Re_{0}$ denote the fiber in $\Re(D)$ at the origin and $A_{0}=\left.H^{\infty}(D)\right|_{\Re_{0}}$.

THEOREM 1. Let $D$ be a $\Delta$-domain with $r_{n} / R_{n} \rightarrow 0$. If $\phi \in \mathbb{T}_{0}$ and $\hat{L}(\phi) \neq$ 0 , then $\phi$ is adherent to $D$.

THEOREM 2. Let $D$ be a $\Delta$-domain. Suppose there exists a positive integer $m$ such that $\Sigma\left(r_{n} / R_{n}\right)^{m}<\infty$. Let $\phi \in \Re_{0}$. If, for some $f \in H^{\infty}(D), \phi(f) \neq 0$ but $a_{n}(f) \rightarrow 0$, then $\phi$ is adherent to $D$.

In [1] Behrens established that $a_{n} \rightarrow \phi_{0}$ in norm if $\Sigma r_{n} / R_{n}<\infty$. Thus Behrens' theorem can be regarded as a consequence of Theorem 2.

\section{Preliminary lemmas and proofs.}

LemMA 1. Let $\varepsilon>0$ be given. Then there exists a positive integer $N$ such that, for all $f \in H^{\infty}(D),\|f\| \leqslant 1$,

$$
\left|f(z)-\left(P_{n} f\right)(z)-a_{n}(f)\right|<\varepsilon, \quad \text { for } z \in B_{n} \backslash \Delta_{n} \text { and } n \geqslant N .
$$

Proof. Choose $N$ so that $S_{n} /\left(R_{n}-S_{n}\right)<\varepsilon, n \geqslant N$. Write $f(z)=\left(P_{n} f\right)(z)$ $+a_{n}(f)+F_{n}(z)$, for $z \in D_{n} \backslash \Delta_{n}$, where

$$
F_{n}(z)=\sum_{j=1}^{\infty} b_{n j}\left(z-c_{n}\right)^{j} \text { and } b_{n j}=\frac{1}{2 \pi i} \int_{\partial D_{n}} \frac{f(\zeta)}{\left(\zeta-c_{n}\right)^{j+1}} d \zeta .
$$

Then $\left|b_{n j}\right| \leqslant 1 / R_{n}^{j}$ and, for $z \in B_{n} \backslash \Delta_{n}, n \geqslant N$,

$$
\left|f(z)-\left(P_{n} f\right)(z)-a_{n}(f)\right|=\left|F_{n}(z)\right| \leqslant \sum_{j=1}^{\infty} \frac{S_{n}^{j}}{R_{n}^{j}}=\frac{S_{n}}{R_{n}-S_{n}}<\varepsilon .
$$

LeMma 2. Let $f_{n} \in H^{\infty}\left(\Delta_{n}^{c}\right)$ with $\left\|f_{n}\right\| \leqslant M, n=1,2, \ldots$ Let

$$
f(z)=\sum_{n=0}^{\infty} L_{n}(z) f_{n}(z)
$$

Then $f \in H^{\infty}(D)$ and, given $\varepsilon>0$, there is a positive integer $N$ such that 


$$
\sum_{n=0 ; n \neq m}^{\infty}\left|L_{n}(z) f_{n}(z)\right|<\varepsilon \text { for } z \in D_{m}, m \geqslant N
$$

Moreover, $a_{n}(f) \rightarrow 0$.

Proof. Observe that $a_{m}(f)=\sum_{n=0 ; n \neq m}^{\infty}\left(L_{n} f_{n}\right)\left(c_{m}\right)$. Incorporating this observation the proof is essentially the same as the analogous lemma in [2].

Define $\Psi: H^{\infty}(D) \rightarrow H^{\infty}\left(\Delta_{0} \times N\right)$ by

$$
\Psi(f)(z, n)=\left(P_{n} f\right) \circ E_{n}^{-1}(z)+a_{n}(f), \quad f \in H^{\infty}(D) .
$$

LEMMA 3. $\Psi$ is continuous.

Proof. An elementary computation shows there exists $M>0$ such that $\left\|P_{n} f\right\|<M\|f\|_{B_{n} \backslash \Delta_{n}}$, for all $n$, whenever $\lim \sup \left(r_{n} / R_{n}\right)<1$.

LEMMA 4. $\|f\|_{\Phi_{0}}=\|\Psi(f)\|_{X}$, all $f \in H^{\infty}(D)$.

Proof. $\|f\|_{\mathscr{N}_{0}}=\lim \sup \|f\|_{B_{n} \backslash \Delta_{n}}=\lim \sup \|\Psi(f)\|_{\Delta_{0} \times\{n\}}=\|\Psi(f)\|_{X}$.

The middle equality follows from Lemma 1 , the left equality from a well-known theorem concerning the Shilov boundary of the fiber algebra [6], and the right equality from the definition of $X$ and elementary properties of $H^{\infty}\left(\Delta_{0} \times N\right)$.

Lemma 5. If $f, g \in H^{\infty}(D)$ then

$$
\left.\Psi(f g)\right|_{X}=\left.\left.\Psi(f)\right|_{X} \cdot \Psi(g)\right|_{X}
$$

Proof. Assume $\|f\| \leqslant 1,\|g\| \leqslant 1$. Let $\varepsilon>0$ be given. Then there exists a positive integer $N$ such that, for $z \in B_{n} \backslash \Delta_{n}, n \geqslant N$,

(1) $\left|f(z)-\left(P_{n} f\right)(z)-a_{n}(f)\right|<\varepsilon / 3$;

(2) $\left|g(z)-\left(P_{n} g\right)(z)-a_{n}(g)\right|<\varepsilon / 3$;

(3) $\left|(f g)(z)-\left(P_{n} f g\right)(z)-a_{n}(f g)\right|<\varepsilon / 3$.

Multiplying (1) and (2) and simplifying we get

$$
\left|-(f g)(z)+\left[\left(P_{n} f\right)(z)+a_{n}(f)\right]\left[\left(P_{n} g\right)(z)+a_{n}(g)\right]\right| \leqslant \varepsilon,
$$

for $z \in B_{n} \backslash \Delta_{n}, n \geqslant N$.

Adding to (3), we get

$$
\left|\left[\left(P_{n} f\right)(z)+a_{n}(f)\right]\left[\left(P_{n} g\right)(z)+a_{n}(g)\right]-\left[\left(P_{n} f g\right)(z)+a_{n}(f g)\right]\right|<2 \varepsilon,
$$

for $z \in B_{n} \backslash \Delta_{n}, n \geqslant N$. Thus

$$
|\Psi(f)(z, n) \cdot \Psi(g)(z, n)-\Psi(f g)(z, n)|<\varepsilon,
$$

for all $z \in \Delta_{0}, n \geqslant N$. It follows that

$$
\Psi(f)(\phi) \cdot \Psi(g)(\phi)=\Psi(f g)(\phi), \quad \text { for } \phi \in X .
$$


Summarizing, we have shown:

Proposition 1. $\Psi$ induces a map $\bar{\Psi}:\left.A_{0} \rightarrow H^{\infty}\left(\Delta_{0} \times N\right)\right|_{X} . \bar{\Psi}$ is an algebra isometric isomorphism of $A_{0}$ with a closed subalgebra $B_{0}$ of $\left.H^{\infty}\left(\Delta_{0} \times N\right)\right|_{X}$.

The map $\theta$ : $\Re\left(B_{0}\right) \rightarrow \Re_{0}$ defined by $\theta(\phi)(f)=\phi(\bar{\Psi}(f))$, for $\phi \in$ $\Re\left(B_{0}\right), f \in A_{0}$, is a homeomorphism. Note that

$$
H^{\infty}\left(\Delta_{0} \times N\right) \cong Z H^{\infty}\left(\Delta_{0} \times N\right)+l^{\infty},
$$

where $Z$ is the "coordinate" function $Z(\lambda, n)=\lambda$. The sequence $\left\{a_{n}(f)\right\}$ is the $l^{\infty}$-component of $\Psi(f)$.

LEMMA 6. $\lim _{n \rightarrow \infty}\left\|f-a_{n}(f)\right\|_{\partial B_{n}}=0$, for $f \in H^{\infty}(D)$.

Proof. From Lemma 1 it suffices to show $\lim _{n \rightarrow \infty}\left\|P_{n} f\right\|_{\partial B_{n}} \rightarrow 0$. But for $z \in \partial B_{n}$,

$$
\left|\left(P_{n} f\right)(z)\right|=\left|\frac{1}{2 \pi i} \int_{\partial \Delta_{n}} \frac{f(\xi)}{\xi-z} d \xi\right| \leqslant\|f\| \frac{r_{n}}{S_{n}-r_{n}} \rightarrow 0 .
$$

LeMma 7. Let $T \in H^{\infty}\left(\Delta_{0} \times N\right)$ be defined by $T(z, n)=z^{k_{n}}$. Then $\bar{\Psi}(L)=$ $T$ and

$$
\left.T H^{\infty}\left(\Delta_{0} \times N\right)\right|_{X}+\mathrm{C} \subset B_{0} .
$$

Proof. Let $h=\left\{z^{k_{n}} h_{n}\right\} \in T H^{\infty}\left(\Delta_{0} \times N\right)$ and let

$$
f=\sum_{n=1}^{\infty} L_{n}\left(h_{n} \circ E_{n}\right)
$$

Then $f \in H^{\infty}(D)$ and $a_{n}(f) \rightarrow 0$, by Lemma 2. Also $\bar{\Psi}(f)=\left.h\right|_{X}$.

At this point recall some general facts. Let $B$ be a commutative Banach algebra, $B_{0}$ a closed subalgebra of $B$, and $J$ a closed ideal in $B$. If $J \subset B_{0}$, then every $\phi \in \mathfrak{T}_{B_{0}}$ which does not annihilate $J$ has a unique extension to $\mathfrak{R}_{B}$. If furthermore, $B_{0}=J+C$, then $\mathfrak{N}_{B_{0}}$ is obtained from $\mathfrak{N}_{B}$ by identifying the hull of $J$ to a point.

If $B_{0}$ is as defined earlier, $B=\left.H^{\infty}\left(\Delta_{0} \times N\right)\right|_{X}$ and $J=\left.T H^{\infty}\left(\Delta_{0} \times N\right)\right|_{X}$, then $\mathfrak{\pi}_{B}=X$ and the following lemma is immediate.

Lemma 8. If $\phi \in \Re\left(B_{0}\right)$ and $\phi(T) \neq 0$, then $\phi$ extends to an evaluation homomorphism at a point of $X$.

For the proof of Theorem 1, we need one final lemma.

LEMMA 9. A homomorphism $\phi \in \Re\left(B_{0}\right)$ is an evaluation homomorphism at a point of $X$ if and only if the corresponding homomorphism $\theta(\phi)$ in $\mathfrak{T}_{0}$ is adherent to $\cup\left(B_{n} \backslash \Delta_{n}\right)$. 
Proof. Suppose $w_{\alpha}$ is a net in $\cup\left(B_{n} \backslash \Delta_{n}\right)$ converging to $\phi \in \Re_{0}$, where $w_{\alpha} \in B_{n_{\alpha}} \backslash \Delta_{n_{\alpha}}$. Define $\left(z_{\alpha}, n_{\alpha}\right) \in \Delta_{0} \times N$ so that $E_{n_{\alpha}}\left(w_{\alpha}\right)=z_{\alpha}$. Then $n_{\alpha} \rightarrow \infty$. Passing to a subnet, we can assume $\left(z_{\alpha}, n_{\alpha}\right)$ converges to $\phi^{*} \in X$. If $f \in$ $H^{\infty}(D)$, then

$$
f(\phi)=\lim f\left(w_{\alpha}\right)=\lim (\Psi f)\left(z_{\alpha}, n_{\alpha}\right) \doteq \Psi f\left(\phi^{*}\right)
$$

so that $\theta\left(\phi^{*}\right)=\phi$.

Conversely, suppose $\phi^{*} \in X$. Since $r_{n} / S_{n} \rightarrow 0, X$ is adherent to

$$
V=U\left[E_{n}\left(B_{n} \backslash \Delta_{n}\right) \times\{n\}\right] \subset \Delta_{0} \times N
$$

Choose a net $\left(z_{\alpha}, n_{\alpha}\right) \in V$ so that $\left(z_{\alpha}, n_{\alpha}\right) \rightarrow \phi^{*}$. Then $n_{\alpha} \rightarrow \infty$. Set $w_{\alpha}=$ $E_{n_{\alpha}}^{-1}\left(z_{\alpha}\right) \in B_{n_{\alpha}} \backslash \Delta_{n_{\alpha}}$. Since $\lim (\Psi f)\left(z_{\alpha}, n_{\alpha}\right)=\Psi f\left(\phi^{*}\right)$ exists for all $f \in$ $H^{\infty}(D)$, also $\lim f\left(w_{\alpha}\right)$ exists. Hence $w_{\alpha}$ converges to some $\phi \in \operatorname{TR}(D)$. Evidently $\phi \in \Re_{0}$ and $\phi=\theta\left(\phi^{*}\right)$.

Proof of Theorem 1. Let $\phi^{*}=\theta^{-1}(\phi)$. Then $\phi^{*}(T)=\phi(L) \neq 0$. By Lemma $8, \phi^{*}$ extends to an evaluation homomorphism at a point of $X$. Thus $\theta\left(\phi^{*}\right)=\phi$ is adherent to $D$ by Lemma 9 .

Corollary 1. D is dense in $\mathfrak{T}(D)$ if and only if $\hat{L}^{-1}(0)$ has no interior.

Note that Lemma 9 also establishes the following proposition:

Proposition 2. Suppose $D$ is a $\Delta$-domain with $r_{n} / R_{n} \rightarrow 0$. If $\mathscr{T}\left(B_{0}\right)$ is the quotient space of $X$ obtained by identifying points not separated by $B_{0}$, then $D$ is dense in $\Re(D)$.

Since the proof of Theorem 1 works for any choice of positive integers $\left\{k_{n}\right\}$ such that

$$
\sum L_{n} f_{n} \in H^{\infty}, \text { for } f_{n} \in H^{\infty}\left(\Delta_{n}^{c}\right),\left\|f_{n}\right\| \leqslant M, n=1,2, \ldots,
$$

one might hope to deduce $D$ is dense in $\Re(D)$ merely under the hypothesis $r_{n} / R_{n} \rightarrow 0$. We have not been able to do this. One difficulty is that the hypothesis $r_{n} / R_{n} \rightarrow 0$ does not guarantee the existence of a distinguished homomorphism in $\Re_{0}$. For example, take $c_{n}=2^{-n}, r_{n}=\varepsilon_{n} 2^{-(n+2)}$ and $R_{n}=$ $2^{-(n+1)}$, where $0<\varepsilon_{n}<1, \varepsilon_{n} \rightarrow 0$ and $\sum \varepsilon_{n}=+\infty$.

Since this is an $(L)$-domain of the type treated by Zalcman in [10] and $\sum r_{n} / c_{n}=\infty$, it follows that there is no distinguished homomorphism in $\mathfrak{\pi}_{0}$. Moreover a theorem of T. Gamelin and J. Garnett [7] shows that in this case the sequence $\left\{a_{n}\right\}$ has a subsequence which is an interpolating sequence.

ProOF OF THEOREM 2. Let $\phi^{*}=\theta^{-1}(\phi), L(z)=\Sigma\left(r_{n} /\left(z-c_{n}\right)\right)^{m}-$ $\left(r_{n} /-c_{n}\right)^{m}$ and $T(z, n)=z^{m}$ so $T=\bar{\Psi}(L)=\left.Z^{m}\right|_{X}$. Then

$$
\left.Z^{m} H^{\infty}\left(\Delta_{0} \times N\right)\right|_{X} \subset B_{0} \text { by Lemma 7, }
$$

where $Z$ denotes the coordinate function in $H^{\infty}\left(\Delta_{0} \times N\right)$. Since $a_{n}(f) \rightarrow$ 
$0,\left.\bar{\Psi}(f) \in Z H^{\infty}\left(\Delta_{0} \times N\right)\right|_{X}$ and $[\bar{\Psi}(f)]^{m}=\left.\bar{\Psi}\left(f^{m}\right) \in Z^{m} H^{\infty}\left(\Delta_{0} \times n\right)\right|_{X}$. Now $0 \neq \phi\left(f^{m}\right)=\phi^{*}\left[\bar{\Psi}\left(f^{m}\right)\right]$. Thus $\phi^{*}$ is not zero on $\left.Z^{m} H^{\infty}\left(\Delta_{0} \times N\right)\right|_{X}$ and $\phi^{*}(T) \neq 0$. By Theorem $1, \phi(L)=\phi^{*}(T) \neq 0$ and $\phi$ is adherent to $D$.

COROLlARY 2. Suppose there exists a positive integer $m$ such that $\Sigma\left(r_{n} / R_{n}\right)^{m}$ $<\infty$ and suppose, for every $f \in H^{\infty}(D), a_{n}(f)$ converges.

Then $D$ is dense in $\Re(D)$.

Proof. Define $\phi_{0}(f)=\lim a_{n}(f), f \in H^{\infty}(D)$. By Lemma 6, $\phi_{0} \in \mathfrak{R}_{0}(D)$ and $\phi_{0}$ is adherent to $D$. If $\phi \in \Re_{0} \backslash\left\{\phi_{0}\right\}$, Theorem 2 shows $\phi$ is adherent to $D$.

We would like to thank the referee for many useful comments and criticisms.

\section{REFERENCES}

1. M. F. Behrens, The corona conjecture for a class of infinitely connected domains, Bull. Amer. Math. Soc. 76 (1970), 387-391. MR 41 \# 825.

2. The maximal ideal space of algebras of bounded analytic functions on infinitely connected domains, Trans. Amer. Math. Soc. 161 (1971), 359-379.

3. L. Carleson, Interpolations by bounded analytic functions and the corona problem, Ann. of Math. (2) 76 (1962), 547-559. MR 25\#5186.

4. T. W. Gamelin, Localization of the corona problem, Pacific J. Math. 34 (1970), 73-81. MR 43\#2482.

5. __ Uniform algebras, Prentice-Hall, Englewood Cliffs, N.J., 1969.

6. Lectures on $H^{\infty}(D)$, La Plata Notas de Math. No. 21 (1972).

7. T. W. Gamelin and J. Garnett, Distinguished homomorphisms and fiber algebras, Amer. J. Math. 92 (1970), 455-474. MR 46 \#2434.

8. K. Hoffman, Banach spaces of analytic functions, Prentice-Hall, Englewood Cliffs, N.J., 1962. MR 24 \# A2844.

9. E. L. Stout, Two theorems concerning functions holomorphic on multiply connected domains, Bull. Amer. Math. Soc. 69 (1963), 527-530. MR 27\#275.

10. L. Zalcman, Bounded analytic functions on domains of infinite connectivity, Trans. Amer. Math. Soc. 144 (1969), 241-269. MR 40 \#5884.

11. W. M. Deeb and D. R. Wilken, $\Delta$-domains and the corona, Trans. Amer. Math. Soc. 231 (1977), 107-115.

Department of Mathematics, State University of New York at Albany, Albany, New YORK 12222 